Journal of Zhejiang University-SCIENCE B (Biomedicine \& Biotechnology) ISSN 1673-1581 (Print); ISSN 1862-1783 (Online)

www.jzus.zju.edu.cn; www.springerlink.com

E-mail: jzus@zju.edu.cn

\title{
Correspondence:
}

\section{Safety of protease inhibitors and Arbidol for SARS-CoV-2 pneumonia in Zhejiang Province, China}

\author{
Yong-zheng GUO, Kai-jin XU, Yong-tao LI, Jia-dan FU, \\ Min XU, Ling YU, Ji-fang SHENG, Biao ZHU ${ }^{\dagger *}$ \\ Department of Infectious Diseases, State Key Laboratory for Diagnosis \\ and Treatment of Infectious Diseases, Collaborative Innovation Center \\ for Diagnosis and Treatment of Infectious Diseases, National Clinical \\ Research Center for Infectious Diseases, the First Affiliated Hospital, \\ Zhejiang University School of Medicine, Hangzhou 310003, China \\ ${ }^{\dagger}$ E-mail: zhubiao1207@zju.edu.cn
}

https://doi.org/10.1631/jzus.B2000204

The aim of this study was to evaluate the safety of an antiviral regimen of protease inhibitors combined with Arbidol (umifenovir) for severe acute respiratory syndrome coronavirus 2 (SARS-CoV-2) pneumonia patients. The genomic sequence of SARS$\mathrm{CoV}-2$ is highly homologous to that of SARS-CoV (Zhou et al., 2020). Previously published basic and clinical research on anti-SARS-CoV treatment found that lopinavir/ritonavir (LPV/r) could improve the prognosis of SARS patients (Chan et al., 2003; Chu et al., 2004). Darunavir (DRV) is another protease inhibitor that blocks the binding of SARS-CoV-2 to human angiotensin-converting enzyme 2 (Omotuyi et al., 2020). The broad-spectrum antiviral drug Arbidol (umifenovir) also shows in vitro anti-SARS-CoV activity (Khamitov et al., 2008).

Therefore, our hospital (the First Affiliated Hospital, Zhejiang University School of Medicine, Hangzhou, China) empirically adopted LPV/r or darunavir/cobicistat (DRV/c) combined with Arbidol as the anti-SARS-

\footnotetext{
"Corresponding author

* Project supported by the National Science and Technology Major Project of China During the 13th Five-Year Plan Period (Nos. 2017ZX 10202102 and 2018ZX10715014)

(1D) ORCID: Yong-zheng GUO, https://orcid.org/0000-0003-4981-8957 (C) Zhejiang University and Springer-Verlag GmbH Germany, part of Springer Nature 2020
}

CoV-2 regimen (National Health Commission of the People's Republic of China, 2020; Xu KJ et al., 2020). Since Jan. 19, 2020, 104 SARS-CoV-2 pneumonia patients have been hospitalized at the First Affiliated Hospital, Zhejiang University School of Medicine. This study is a retrospective analysis of the clinical data of 52 of these patients to evaluate the safety of the antiviral regimen of protease inhibitors combined with Arbidol; this could provide evidence to further improve anti-SARS-CoV-2 therapy.

All SARS-CoV-2 pneumonia patients who were cured and discharged from the Department of Infectious Diseases of the First Affiliated Hospital, Zhejiang University School of Medicine between Jan. 19 and Mar. 5, 2020, and who met the inclusion criteria of this study, were included in our analysis. The inclusion criteria were all of the following: real-time reverse transcription-polymerase chain reaction (RT-qPCR)based detection of SARS-CoV-2 nucleic acid in sputum; computed tomography (CT) imaging features of SARS-CoV-2 pneumonia; antiviral therapy including protease inhibitors and Arbidol. DRV instead of LPV was chosen for SARS-CoV-2 pneumonia patients with abnormally elevated lipid levels on admission. The exclusion criteria were as follows: pregnant patients; patients who did not receive protease inhibitors or Arbidol; patients who received protease inhibitors or Arbidol for less than $3 \mathrm{~d}$; patients who were on statins or fibrate before the onset of illness; patients with missing data at the baseline or before discharge; patients who were transferred to our hospital after being hospitalized elsewhere for more than $3 \mathrm{~d}$; patients who received invasive mechanical ventilation, extracorporeal membrane oxygenation, or blood purification during hospitalization. This study was approved by the Ethics Committee of the First Affiliated Hospital, Zhejiang University School of Medicine. 
We performed a retrospective analysis of clinical symptoms, liver function, and lipid profile data of SARS-CoV-2 pneumonia patients at admission and before discharge. The changes in symptoms before and after treatment, liver function and blood lipid levels, and proportion of patients with abnormal indicators were noted. The Self-Reporting Questionnaire-20 (SRQ-20), 9-Item Patient Health Questionnaire (PHQ-9), Generalized Anxiety Disorder 7-Item (GAD-7), Pittsburgh Sleep Quality Index (PSQI), Hamilton Depression Scale (HAMD), Hamilton Anxiety Scale (HAMA), and Positive and Negative Syndrome Scale (PANSS) were used to evaluate the psychological symptoms of patients.

SPSS 23.0 statistical software was used to analyze the clinical data. Count data were expressed using number of cases (percentage), and the chi-square test was used to compare groups. Non-normal distribution measurement data were expressed as median and interquartile range $(M(\mathrm{IQR}))$. Mann-Whitney $U$-test was performed to compare groups. $P<0.05$ was considered statistically significant.

Forty-three (82.7\%) patients received LPV/r as the initial antiviral regimen, whereas six patients were changed to DRV/c due to obvious nausea, diarrhoea, and other gastrointestinal symptoms after using LPV/r. Nine patients were initially selected to receive DRV/c rather than LPV/r because of abnormally elevated lipid levels at admission. A total of 37 patients $(71.2 \%)$ received glucocorticoids orally or intravenously (Table 1).

The numbers of patients with diarrhea $(10,19.2 \%)$, reduced food intake $(9,17.3 \%)$, constipation (7,
$13.5 \%)$, and psychological abnormalities $(19,36.5 \%)$ increased significantly after treatment (Table 2). Psychological abnormalities included sleep disturbance $(15,28.9 \%)$, anxiety $(4,7.7 \%)$, and depression $(1,1.9 \%)$. Serum triglyceride (TG), total cholesterol (TC), and low-density lipoprotein (LDL) levels, as well as abnormal indicators, were significantly higher in SARSCoV-2 pneumonia patients after treatment (all $P<0.01$ ) than before. Serum levels of high-density lipoprotein (HDL) increased significantly and TC/HDL ratio decreased significantly after treatment (all $P<0.01$ ). Twenty-two patients (42.3\%) had elevated levels of alanine aminotransferase (ALT) or aspartate aminotransferase (AST) during hospitalization, but no serious liver damage was observed. Moreover, serum AST levels were significantly lower after treatment $(P<0.01$; Table 2).

Thirty-seven patients in the LPV/r group had significantly higher serum TG levels and abnormal indicators post-treatment, and the number of patients with abnormal LDL levels was also significantly higher. Four of the nine patients who initially chose $\mathrm{DRV} / \mathrm{c}$ reported constipation after treatment ( 0 vs. 4 , $44.4 \%, P=0.023$; Table 3).

There were 15 patients in the DRV/c group, of which 10 patients received glucocorticoids; 37 patients in the LPV/r group, of which 27 patients received glucocorticoids. There was no significant difference in demographic or epidemiologic data of the two patient groups at baseline. The Mann-Whitney $U$-test was performed on the glucocorticoid usage of the two groups $(P=0.42)$.

Table 1 Demographic and epidemiologic data of the patients at baseline

\begin{tabular}{llll}
\hline \multicolumn{1}{c}{ Characteristics } & \multicolumn{1}{c}{ Total $(n=52)$} & \multicolumn{1}{c}{ LPV/r $(n=37)$} & DAR/c $(n=15)$ \\
\hline Male & $30(57.7 \%)$ & $23(62.2 \%)$ & $7(46.7 \%)$ \\
Female & $22(42.3 \%)$ & $14(37.8 \%)$ & $8(53.3 \%)$ \\
Age (year) & $52.50(44.25,60.00)$ & $51.00(38.50,60.00)$ & $53.00(46.00,65.00)$ \\
Days in hospital (d) & $17.00(12.25,22.75)$ & $17.00(11.50,22.50)$ & $19.00(14.00,24.00)$ \\
Coexisting condition & $27(51.9 \%)$ & $19(51.4 \%)$ & $8(53.3 \%)$ \\
Hypertension & $13(25.0 \%)$ & $7(18.9 \%)$ & $6(40.0 \%)$ \\
Fatty liver disease & $15(28.8 \%)$ & $10(27.0 \%)$ & $5(33.3 \%)$ \\
Diabetes & $6(11.5 \%)$ & $5(13.5 \%)$ & $1(6.7 \%)$ \\
Chronic viral hepatitis B & $3(5.8 \%)$ & $3(8.1 \%)$ & 0 \\
Chronic obstructive pulmonary disease & $2(3.8 \%)$ & $2(5.4 \%)$ & 0 \\
Antiviral drug (d) & $12.50(8.25,17.00)$ & $11.00(8.00,16.50)$ & $14.00(8.00,19.00)$ \\
\hline
\end{tabular}

Data are expressed as number (percentage) of cases or median (interquartile range) (M (IQR)). DRV/c, darunavir/cobicistat; LPV/r, lopinavir/ritonavir 
Table 2 Changes in clinical symptoms, liver function, and serum lipids in 52 SARS-CoV-2 pneumonia patients before and after treatment

\begin{tabular}{|c|c|c|c|}
\hline Characteristics & Before & After & $P$-value \\
\hline \multicolumn{4}{|l|}{ Symptom } \\
\hline Fever & $40(76.9 \%)$ & $3(5.8 \%)$ & $<0.001$ \\
\hline Cough & $42(80.8 \%)$ & $40(76.9 \%)$ & 0.631 \\
\hline Sputum production & $24(46.2 \%)$ & $26(50.0 \%)$ & 0.695 \\
\hline Muscle aches & $11(21.2 \%)$ & $10(19.2 \%)$ & 0.807 \\
\hline Shortness of breath & $9(17.3 \%)$ & $15(28.9 \%)$ & 0.163 \\
\hline Fatigue & $8(15.4 \%)$ & $10(19.2 \%)$ & 0.604 \\
\hline Nausea & $3(5.8 \%)$ & $7(13.5 \%)$ & 0.183 \\
\hline Vomiting & 0 & $5(9.6 \%)$ & 0.057 \\
\hline Reduced food intake & $2(3.9 \%)$ & $9(17.3 \%)$ & 0.026 \\
\hline Diarrhea & $1(1.9 \%)$ & $10(19.2 \%)$ & 0.004 \\
\hline Headache & $1(1.9 \%)$ & $4(7.7 \%)$ & 0.363 \\
\hline Constipation & 0 & $7(13.5 \%)$ & 0.006 \\
\hline Rash & 0 & $3(5.8 \%)$ & 0.243 \\
\hline Itching & 0 & $1(1.9 \%)$ & 1.000 \\
\hline Psychological abnormalities & 0 & $19(36.5 \%)$ & $<0.001$ \\
\hline \multicolumn{4}{|l|}{ Liver function } \\
\hline ALT (U/L) & $23.00(15.00,37.75)$ & $28.50(17.00,45.50)$ & 0.094 \\
\hline$>50 \mathrm{U} / \mathrm{L}$ & $8(15.4 \%)$ & $10(19.2 \%)$ & 0.604 \\
\hline AST (U/L) & $20.00(18.00,30.75)$ & $15.50(13.00,21.00)$ & $<0.001$ \\
\hline$>40 \mathrm{U} / \mathrm{L}$ & $10(19.2 \%)$ & $3(5.8 \%)$ & 0.038 \\
\hline $\mathrm{TB}(\mu \mathrm{mol} / \mathrm{L})$ & $10.95(7.23,16.88)$ & $9.15(6.93,12.28)$ & 0.050 \\
\hline$>26 \mu \mathrm{mol} / \mathrm{L}$ & $6(11.5 \%)$ & $1(1.9 \%)$ & 0.112 \\
\hline $\operatorname{ALP}(\mathrm{U} / \mathrm{L})$ & $61.50(53.00,75.00)$ & $66.00(55.25,76.50)$ & 0.537 \\
\hline$>125 \mathrm{U} / \mathrm{L}$ & $1(1.9 \%)$ & $1(1.9 \%)$ & 1.000 \\
\hline GGT (U/L) & $28.00(17.50,45.00)$ & $30.50(22.25,58.75)$ & 0.204 \\
\hline$>60 \mathrm{U} / \mathrm{L}$ & $9(17.3 \%)$ & $12(23.1 \%)$ & 0.464 \\
\hline \multicolumn{4}{|l|}{ Serum lipid } \\
\hline TG (mol/L) & $1.10(0.83,1.48)$ & $1.89(1.41,2.88)$ & $<0.001$ \\
\hline$\geq 1.70 \mathrm{~mol} / \mathrm{L}$ & $11(21.2 \%)$ & $33(63.5 \%)$ & $<0.001$ \\
\hline $\mathrm{TC}(\mathrm{mol} / \mathrm{L})$ & $3.87(3.33,4.17)$ & $4.49(4.04,5.44)$ & $<0.001$ \\
\hline$\geq 5.18 \mathrm{~mol} / \mathrm{L}$ & $2(3.9 \%)$ & $16(30.8 \%)$ & $<0.001$ \\
\hline HDL (mol/L) & $0.95(0.83,1.13)$ & $1.14(0.96,1.44)$ & 0.001 \\
\hline$<1.04 \mathrm{~mol} / \mathrm{L}$ & $30(57.7 \%)$ & $20(38.5 \%)$ & 0.039 \\
\hline $\mathrm{LDL}(\mathrm{mol} / \mathrm{L})$ & $2.18(1.80,2.56)$ & $3.98(3.35,4.87)$ & $<0.001$ \\
\hline$\geq 3.37 \mathrm{~mol} / \mathrm{L}$ & $1(1.9 \%)$ & $10(19.2 \%)$ & 0.005 \\
\hline TC/HDL & $3.68(3.10,4.74)$ & $2.63(2.14,3.26)$ & $<0.001$ \\
\hline$\geq 5$ & $8(15.4 \%)$ & $13(25.0 \%)$ & 0.241 \\
\hline
\end{tabular}

Data are expressed as number (percentage) of cases or median (interquartile range) ( $M(\mathrm{IQR}))$. ALT, alanine aminotransferase; ALP, alkaline phosphatase; AST, aspartate aminotransferase; LDL, low-density lipoprotein; GGT, glutamyl transpeptidase; HDL, high-density lipoprotein; SARS-CoV-2, severe acute respiratory syndrome coronavirus 2; TB, total bilirubin; TC, total cholesterol; TG, triglyceride

Regardless of whether the treatment regimen included glucocorticoids, the TG level and the number of patients with abnormal TG levels increased significantly post-treatment. Moreover, no significant differences were observed in the TG levels between the glucocorticoid-treated group and the untreated group. Serum TC and LDL levels were significantly higher in both glucocorticoid-treated and untreated patients. After treatment, the serum HDL level and the number of patients with abnormal serum TC 
Table 3 Changes in gastrointestinal symptoms, liver function, and serum lipids in the LPV/r and DRV/c groups before and after treatment

\begin{tabular}{|c|c|c|c|c|c|c|}
\hline \multirow{2}{*}{ Characteristics } & \multicolumn{3}{|c|}{$\mathrm{LPV} / \mathrm{r}(n=37)$} & \multicolumn{3}{|c|}{$\mathrm{DAR} / \mathrm{c}(n=15)$} \\
\hline & Before & After & $P$-value & Before & After & $P$-value \\
\hline \multicolumn{7}{|l|}{ Gastrointestinal symptom } \\
\hline Nausea $^{*}$ & $2(5.4 \%)$ & $4(10.8 \%)$ & 0.394 & 0 & 0 & \\
\hline Vomiting $^{*}$ & 0 & $2(5.4 \%)$ & 0.152 & 0 & 0 & \\
\hline Reduced food intake ${ }^{*}$ & $2(5.4 \%)$ & $6(16.2 \%)$ & 0.134 & 0 & $1(11.1 \%)$ & 0.303 \\
\hline Diarrhea $^{*}$ & $1(2.7 \%)$ & $4(10.8 \%)$ & 0.165 & 0 & $1(11.1 \%)$ & 0.303 \\
\hline Constipation $^{*}$ & 0 & $2(5.4 \%)$ & 0.152 & 0 & $4(44.4 \%)$ & 0.023 \\
\hline \multicolumn{7}{|l|}{ Liver function } \\
\hline $\operatorname{ALT}(\mathrm{U} / \mathrm{L})$ & $24.0(15.5,37.5)$ & $29.0(16.0,56.0)$ & 0.261 & $21.0(13.0,39.0)$ & $28.0(22.0,33.0)$ & 0.305 \\
\hline$>50 \mathrm{U} / \mathrm{L}$ & $7(18.9 \%)$ & $9(24.3 \%)$ & 0.572 & $1(6.7 \%)$ & $1(6.7 \%)$ & 1.000 \\
\hline $\operatorname{AST}(\mathrm{U} / \mathrm{L})$ & $21.0(18.0,32.5)$ & $17.0(13.5,23.5)$ & 0.003 & $18.0(15.0,30.0)$ & $14.0(12.0,18.0)$ & 0.011 \\
\hline$>40 \mathrm{U} / \mathrm{L}$ & $7(18.9 \%)$ & $3(8.1 \%)$ & 0.174 & $3(20.0 \%)$ & 0 & 0.068 \\
\hline $\mathrm{TB}(\mu \mathrm{mol} / \mathrm{L})$ & $11.1(8.5,17.6)$ & $10.1(7.6,12.9)$ & 0.147 & $10.1(5.4,13.7)$ & $6.9(4.0,10.6)$ & 0.067 \\
\hline$>26 \mu \mathrm{mol} / \mathrm{L}$ & $5(13.5 \%)$ & $1(2.7 \%)$ & 0.088 & $1(6.7 \%)$ & 0 & 0.309 \\
\hline $\operatorname{ALP}(\mathrm{U} / \mathrm{L})$ & $62.0(53.5,75.0)$ & $63.0(55.5,75.5)$ & 0.875 & $58.0(50.0,77.0)$ & $73.0(54.0,77.0)$ & 0.486 \\
\hline$>125 \mathrm{U} / \mathrm{L}$ & $1(2.7 \%)$ & 0 & 0.314 & 0 & $1(6.7 \%)$ & 0.309 \\
\hline GGT (U/L) & $28.0(20.0,55.5)$ & $39.0(20.5,82.0)$ & 0.328 & $29.0(16.0,35.0)$ & $29.0(24.0,34.0)$ & 0.624 \\
\hline$>60 \mathrm{U} / \mathrm{L}$ & $8(21.6 \%)$ & $12(32.4 \%)$ & 0.295 & $1(6.7 \%)$ & 0 & 0.309 \\
\hline \multicolumn{7}{|l|}{ Serum lipid } \\
\hline TG (mol/L) & $1.09(0.74,1.44)$ & $2.14(1.59,3.07)$ & $<0.001$ & $1.20(1.10,1.76)$ & $1.44(1.05,2.43)$ & 0.290 \\
\hline$\geq 1.70 \mathrm{~mol} / \mathrm{L}$ & $6(16.2 \%)$ & $26(70.3 \%)$ & $<0.001$ & $5(33.3 \%)$ & $7(46.7 \%)$ & 0.550 \\
\hline $\mathrm{TC}(\mathrm{mol} / \mathrm{L})$ & $3.70(3.29,4.04)$ & $4.32(3.91,5.18)$ & $<0.001$ & $4.03(3.50,4.66)$ & $5.14(4.31,5.48)$ & 0.003 \\
\hline$\geq 5.18 \mathrm{~mol} / \mathrm{L}$ & $1(2.7 \%)$ & $9(24.3 \%)$ & 0.007 & $1(6.7 \%)$ & $7(46.7 \%)$ & 0.017 \\
\hline HDL (mol/L) & $0.97(0.83,1.18)$ & $1.13(0.96,1.47)$ & 0.019 & $0.95(0.83,1.04)$ & $1.16(0.99,1.30)$ & 0.006 \\
\hline$<1.04 \mathrm{~mol} / \mathrm{L}$ & $21(56.8 \%)$ & $15(40.5 \%)$ & 0.163 & $9(60.0 \%)$ & $5(33.3 \%)$ & 0.096 \\
\hline LDL (mol/L) & $2.04(1.73,2.45)$ & $4.06(3.24,4.94)$ & $<0.001$ & $2.35(1.96,2.94)$ & $3.92(3.52,4.79)$ & 0.000 \\
\hline$\geq 3.37 \mathrm{~mol} / \mathrm{L}$ & 0 & $6(16.2 \%)$ & 0.010 & $1(6.7 \%)$ & $4(26.7 \%)$ & 0.164 \\
\hline $\mathrm{TC} / \mathrm{HDL}$ & $3.45(2.98,4.61)$ & $2.39(1.96,2.98)$ & $<0.001$ & $4.33(3.49,4.98)$ & $3.15(2.64,3.94)$ & 0.009 \\
\hline$\geq 5$ & $5(13.5 \%)$ & $8(21.6 \%)$ & 0.359 & $3(20.0 \%)$ & $5(33.3 \%)$ & 0.474 \\
\hline
\end{tabular}

Data are expressed as number (percentage) of cases or median (interquartile range) (M (IQR)). ${ }^{*}$ Only the clinical symptoms of nine patients who initially adopted DRV/c were analyzed. ALP, alkaline phosphatase; ALT, alanine aminotransferase; AST, aspartate aminotransferase; DRV/c, darunavir/cobicistat; GGT, glutamyl transpeptidase; HDL, high-density lipoprotein; LDL, low-density lipoprotein; LPV/r, lopinavir/ ritonavir; TB, total bilirubin; TC, total cholesterol; TG, triglyceride

and LDL levels in the glucocorticoid group were significantly higher, whereas the proportion of patients with abnormal HDL levels was significantly lower (Table 4).

The present retrospective analysis shows that although combinations of these antiviral drugs, namely $\mathrm{LPV} / \mathrm{r}$ or DRV/c with Arbidol, can result in viral nucleic acid-negative tests in sputum by $12-14 \mathrm{~d}$ after the initiation of treatment, they significantly affect lipid metabolism in SARS-CoV-2 pneumonia patients, with the negative impact of DRV/c being less than that of LPV/r. There are no antiviral drugs specifically developed for use against SARS-CoV-2. Based on previous studies on SARS-CoV, especially in vitro studies, we adopted the early use of protease inhibitors (LPV/r or DRV/c) combined with Arbidol as our antiviral strategy (Chan et al., 2003; Chu et al., 2004; Khamitov et al., 2008; Omotuyi et al., 2020). Coronavirus disease 2019 (COVID-19) patients who underwent this antiviral therapy first tested negative for SARS-CoV-2 nucleic acid in sputum after an average of $12.0(8.0,15.0) \mathrm{d}$ of treatment, and tested negative continuously after an average of $13.5(9.5,17.5) \mathrm{d}(\mathrm{Xu}$ $\mathrm{KJ}$ et al., 2020). LPV/r is currently the most widely 
Table 4 Blood lipid levels and abnormal indicators before and after treatment based on patient's glucocorticoid use

\begin{tabular}{|c|c|c|c|c|c|c|c|c|}
\hline \multirow{2}{*}{ Characteristics } & \multicolumn{3}{|c|}{ Glucocorticoids-untreated $(n=15)$} & \multicolumn{3}{|c|}{ Glucocorticoids-treated $(n=37)$} & \multicolumn{2}{|c|}{$P$-value } \\
\hline & Before & After & $P$-value & Before & After & $P$-value & Before $^{*}$ & After $^{\#}$ \\
\hline TG (mol/L) & $\begin{array}{c}1.08 \\
(0.60,1.73)\end{array}$ & $\begin{array}{c}2.61 \\
(1.44,4.20)\end{array}$ & 0.001 & $\begin{array}{c}1.10 \\
(0.86,1.47)\end{array}$ & $\begin{array}{c}1.83 \\
(1.36,2.49)\end{array}$ & $<0.001$ & 0.316 & 0.110 \\
\hline$\geq 1.70 \mathrm{~mol} / \mathrm{L}$ & $4(26.7 \%)$ & $10(66.7 \%)$ & 0.028 & $7(19.4 \%)$ & $23(62.2 \%)$ & $<0.001$ & 0.568 & 0.760 \\
\hline $\mathrm{TC}(\mathrm{mol} / \mathrm{L})$ & $\begin{array}{c}3.93 \\
(3.55,4.17)\end{array}$ & $\begin{array}{c}4.32 \\
(3.81,5.14)\end{array}$ & 0.041 & $\begin{array}{c}3.70 \\
(3.14,4.20)\end{array}$ & $\begin{array}{c}4.67 \\
(4.13,5.60)\end{array}$ & $<0.001$ & 0.260 & 0.110 \\
\hline$\geq 5.18 \mathrm{~mol} / \mathrm{L}$ & 0 & $2(13.3 \%)$ & 0.143 & $2(5.6 \%)$ & $14(37.8 \%)$ & 0.001 & 0.352 & 0.760 \\
\hline $\mathrm{HDL}(\mathrm{mol} / \mathrm{L})$ & $\begin{array}{c}1.10 \\
(0.84,1.28)\end{array}$ & $\begin{array}{c}0.90 \\
(0.86,1.05)\end{array}$ & 0.436 & $\begin{array}{c}0.93 \\
(0.83,1.08)\end{array}$ & $\begin{array}{c}1.20 \\
(1.03,1.49)\end{array}$ & $<0.001$ & 0.163 & 0.001 \\
\hline$<1.04 \mathrm{~mol} / \mathrm{L}$ & $6(40.0 \%)$ & $10(66.7 \%)$ & 0.143 & $24(66.7 \%)$ & $10(27.0 \%)$ & 0.001 & 0.078 & 0.008 \\
\hline $\mathrm{LDL}(\mathrm{mol} / \mathrm{L})$ & $\begin{array}{c}2.22 \\
(1.99,2.46)\end{array}$ & $\begin{array}{c}4.60 \\
(3.84,5.43)\end{array}$ & $<0.001$ & $\begin{array}{c}2.11 \\
(1.72,2.61)\end{array}$ & $\begin{array}{c}3.77 \\
(3.21,4.43)\end{array}$ & $<0.001$ & 0.414 & 0.036 \\
\hline$\geq 3.37 \mathrm{~mol} / \mathrm{L}$ & 0 & $2(13.3 \%)$ & 0.143 & $1(2.8 \%)$ & $8(21.6 \%)$ & 0.014 & 0.514 & 0.492 \\
\hline TC/HDL & $\begin{array}{c}3.57 \\
(2.82,4.71)\end{array}$ & $\begin{array}{c}2.39 \\
(1.87,3.22)\end{array}$ & 0.005 & $\begin{array}{c}3.69 \\
(3.13,4.83)\end{array}$ & $\begin{array}{c}2.69 \\
(2.16,3.30)\end{array}$ & $<0.001$ & 0.591 & 0.257 \\
\hline$\geq 5$ & $2(13.3 \%)$ & $7(46.7 \%)$ & 0.046 & $6(16.7 \%)$ & $6(16.2 \%)$ & 0.959 & 0.766 & 0.022 \\
\hline
\end{tabular}

Data are expressed as number (percentage) of cases or median (interquartile range) (M(IQR)). ${ }^{*}$ Mann-Whitney $U$-test and chi-square test results of blood lipid levels and abnormal indicators of glucocorticoid-treated and untreated groups before treatment; ${ }^{\#}$ Mann-Whitney $U$-test and chi-square test results of blood lipid levels and abnormal indicators of glucocorticoid-treated and untreated groups after treatment. LDL, low-density lipoprotein; HDL, high-density lipoprotein; TC, total cholesterol; TG, triglyceride

used anti-human immunodeficiency viral (HIV) protease inhibitor drug and is well tolerated by patients. The main adverse events are gastrointestinal distress, usually diarrhea, and abnormal lipid metabolism, principally elevated TG and TC levels. Some patients may also have abnormal serum transaminase, LDL, or HDL levels (Orkin et al., 2013; Huang et al., 2019). Arbidol is a broad-spectrum antiviral drug that mainly shows efficacy against influenza viruses. Its adverse events are mainly gastrointestinal distress and elevated serum ALT levels (Wang et al., 2004). Therefore, our study focused principally on the adverse effects of our antiviral strategy with respect to gastrointestinal symptoms, liver function, and serum lipids in SARS-CoV-2 pneumonia patients.

In our study we found that the most significant clinical symptoms of SARS-CoV-2 pneumonia patients during hospitalization were psychological abnormalities, followed by gastrointestinal distress. Evaluation by psychologists revealed that approximately half of the hospitalized patients showed different degrees of psychological abnormalities (Xu KJ et al., 2020). This might be related to the psychological stress of being diagnosed with SARS-CoV-2 infection and isolated for treatment. After psychotherapy with or without medication, these symptoms improved significantly in approximately $82.3 \%$ of the patients
(Xu KJ et al., 2020). In another study on LPV/r antiSARS-CoV therapy, 11 patients $(26.83 \%)$ experienced gastrointestinal distress (Chu et al., 2004). We speculated that in addition to the antiviral drugs, the patients' gastrointestinal distress could also be attributed to reduced physical activity, emotional abnormalities, and treatment with psychiatric drugs. The negative effects of DRV on the gastrointestinal tract are less than those of LPV (Ortiz et al., 2008; Banhegyi et al., 2012; Orkin et al., 2013). In the current study, six patients $(11.5 \%)$ experienced nausea and/or diarrhea after receiving LPV/r; however, the replacement of $\mathrm{LPV} / \mathrm{r}$ with DRV/c and symptomatic treatment significantly alleviated the patients' gastrointestinal discomfort.

A multi-center clinical study of HIV-infected patients in China treated with LPV/r found that only one patient had abnormal liver function (Huang et al., 2019). In a clinical study of LPV/r combined with ribavirin for the treatment of SARS, hepatic injury was observed in seven patients (17.07\%) (Chu et al., 2004). In our previous study, 10 (16\%) patients with SARS-CoV-2 infection had abnormally elevated serum AST levels (Xu XW et al., 2020). In our current study, $15.4 \%$ and $19.2 \%$ of patients had elevated levels of ALT and AST, respectively. Although some patients had elevated levels of transaminase during their hospitalization, there were no discontinuation of 
therapy due to liver injury. Additionally, the serum AST levels and proportion of patients with abnormal AST levels were significantly lower after treatment.

The study of Huang et al. (2019) on LPV/rbased anti-HIV therapy in Chinese patients have found that LPV/r-related lipid metabolism abnormalities mainly present as markedly elevated TC and TG levels, whereas LDL levels are normal. In the present study, serum TG, TC, and LDL levels, and the proportion of patients with abnormal levels of these indicators were significantly higher after treatment.

Increased LDL levels are crucial early indicators of atherosclerotic cardiovascular disease (National Cholesterol Education Program (NCEP) Expert Panel on Detection, Evaluation, and Treatment of High Blood Cholesterol in Adults (Adult Treatment Panel III), 2002). In HIV populations, patients receiving DRV have lower serum TG and TC levels than patients receiving LPV (Ortiz et al., 2008; Banhegyi et al. 2012; Orkin et al., 2013). Therefore, we recommend that all discharged patients should be monitored for changes in blood lipid levels and provide timely assessment of the risk of ischemic cardiovascular disease.

Seventy-eight (75.0\%) SARS-CoV-2 pneumonia patients hospitalized in our center were seriously/ critically ill. To control the cytokine storm, these patients were treated with short-course glucocorticoids in the early stage of the disease (National Health Commission of the People's Republic of China, 2020; $\mathrm{Xu} \mathrm{KJ}$ et al., 2020). However, glucocorticoids can affect lipid metabolism (Ramshanker et al., 2019). We found that the serum TC, TG, and LDL levels in patients increased, regardless of the use of glucocorticoids.

Our study has several limitations. In particular, this is a single-center retrospective study that lacks randomized controls. We did not evaluate QT prolongation, myelosuppression, or CYP3A inhibitioninduced multiple drug interactions. The lack of follow-up data after discharge from the hospital might also mean a lack of important information that could affect the safety assessment of the antiviral regimen.

In our retrospective study, we found that an antiviral regimen consisting of a combination of protease inhibitors and Arbidol can significantly affect lipid metabolism in SARS-CoV-2 pneumonia patients, especially TG, TC, and LDL levels. DRV/c has a lower negative impact on lipid metabolism compared with LPV/r. The most common clinical abnormalities associated with antiviral therapy are diarrhea, constipation, and decreased food intake. Patients tolerated treatment well after changing the antiviral drug from $\mathrm{LPV} / \mathrm{r}$ to DRV/c and receiving other corresponding symptomatic treatments. In order to arrive at more credible conclusions, further randomized double-blind prospective studies are needed to evaluate the efficacy and safety of these medications for the treatment of SARS-CoV-2 infection.

\section{Contributors}

Kai-jin XU and Biao ZHU designed the study. Yongzheng GUO coordinated the work, developed the statistical methods, and took the lead in drafting the manuscript and interpreting. Yong-tao LI participated in the data analysis. Jia-dan FU, Min XU, and Ling YU performed the data collection. Ji-fang SHENG participated in writing and editing of the manuscript. The corresponding author attests that all listed authors meet authorship criteria and that no others meeting the criteria have been omitted. All authors have read and approved the final manuscript, and therefore, have full access to all the data in the study and take responsibility for the integrity of the data and the accuracy of the data analysis.

\section{Acknowledgments}

All authors participated in the fight against SARS-CoV-2 on the front line, and we especially acknowledge and honor all medical personnel who sacrificed their lives in this battle.

\section{Compliance with ethics guidelines}

Yong-zheng GUO, Kai-jin XU, Yong-tao LI, Jia-dan FU, Min XU, Ling YU, Ji-fang SHENG, and Biao ZHU declare that they have no conflict of interest.

All procedures followed were in accordance with the ethical standards of the responsible committee on human experimentation (institutional and national) and with the Helsinki Declaration of 1975, as revised in 2008(5). Informed consent was obtained from all patients for being included in the study.

\section{References}

Banhegyi D, Katlama C, da Cunha CA, et al., 2012. Week 96 efficacy, virology and safety of darunavir/r versus lopinavir/r in treatment-experienced patients in TITAN. Curr HIV Res, 10(2):171-181. https://doi.org/10.2174/157016212799937218

Chan KS, Lai ST, Chu CM, et al., 2003. Treatment of severe acute respiratory syndrome with lopinavir/ritonavir: a multicentre retrospective matched cohort study. Hong Kong Med J, 9(6):399-406.

Chu CM, Cheng VCC, Hung IFN, et al., 2004. Role of lopinavir/ ritonavir in the treatment of SARS: initial virological and clinical findings. Thorax, 59(3):252-256. 
https://doi.org/10.1136/thorax.2003.012658

Huang XJ, Xu LM, Sun LJ, et al., 2019. Six-year immunologic recovery and virological suppression of HIV patients on LPV/r-based second-line antiretroviral treatment: a multicenter real-world cohort study in China. Front Pharmacol, 10:1455. https://doi.org/10.3389/fphar.2019.01455

Khamitov RA, Loginova SI, Shchukina VN, et al., 2008. Antiviral activity of arbidol and its derivatives against the pathogen of severe acute respiratory syndrome in the cell cultures. Vopr Virusol, 53(4):9-13.

National Cholesterol Education Program (NCEP) Expert Panel on Detection, Evaluation, and Treatment of High Blood Cholesterol in Adults (Adult Treatment Panel III), 2002. Third report of the National Cholesterol Education Program (NCEP) Expert Panel on Detection, Evaluation, and Treatment of High Blood Cholesterol in Adults (Adult Treatment Panel III) final report. Circulation, 106(25): 3143-3421.

https://doi.org/10.1161/circ.106.25.3143

National Health Commission of the People's Republic of China, 2020. Diagnosis and treatment of novel coronavirus pneumonia (Trial Version 7) (in Chinese). http://www. nhc.gov.cn/xcs/zhengcwj/202003/46c9294a7dfe4cef80dc7 f5912eb1989.shtml [Accessed on Mar. 11, 2020].

Omotuyi OI, Nash O, Ajiboye BO, et al., 2020. Darunavir disrupts critical nodes in metastable 2019-nCoV-RBD/ ACE-2 complex. Preprints, 2020030125. https://doi.org/10.20944/preprints202003.0125.v1

Orkin C, DeJesus E, Khanlou H, et al., 2013. Final 192-week efficacy and safety of once-daily darunavir/ritonavir compared with lopinavir/ritonavir in HIV-1-infected treatmentnaive patients in the ARTEMIS trial. HIV Med, 14(1): 49-59.

https://doi.org/10.1111/j.1468-1293.2012.01060.x

Ortiz R, Dejesus E, Khanlou H, et al., 2008. Efficacy and safety of once-daily darunavir/ritonavir versus lopinavir/ ritonavir in treatment-naive HIV-1-infected patients at week 48. AIDS, 22(12):1389-1397. https://doi.org/10.1097/QAD.0b013e32830285fb

Ramshanker N, Jessen N, Voss TS, et al., 2019. Effects of short-term prednisolone treatment on indices of lipolysis and lipase signaling in abdominal adipose tissue in healthy humans. Metabolism, 99:1-10. https://doi.org/10.1016/j.metabol.2019.06.013

Wang MZ, Cai BQ, Li LY, et al., 2004. Efficacy and safety of Arbidol in treatment of naturally acquired influenza. Acta Acad Med Sin, 26(3):289-293 (in Chinese).

$\mathrm{Xu} \mathrm{KJ}$, Cai HL, Shen YH, et al., 2020. Management of COVID-19: the Zhejiang experience. J Zhejiang Univ (Med Sci), 49(2):147-157 (in Chinese). https://doi.org/10.3785/j.issn.1008-9292.2020.02.02

$\mathrm{Xu}$ XW, Wu XX, Jiang XG, et al., 2020. Clinical findings in a group of patients infected with the 2019 novel coronavirus (SARS-CoV-2) outside of Wuhan, China: retrospective case series. BMJ, 368:m606. https://doi.org/10.1136/bmj.m606

Zhou P, Yang XL, Wang XG, et al., 2020. A pneumonia outbreak associated with a new coronavirus of probable bat origin. Nature, 579(7798):270-273.

https://doi.org/10.1038/s41586-020-2012-7

\section{中文概要}

\section{题 目: 蛋白酶抑制剂联合阿比多尔的抗病毒方案在浙江 省 SARS-CoV-2 肺炎患者中的安全性研究}

目 的: 评价蛋白酶抑制剂联合阿比多尔的抗病毒方案在 严重急性呼吸综合征冠状病毒 2（SARS-CoV-2） 肺炎患者中应用的安全性。

创新点: 首次评价了蛋白酶抑制剂联合阿比多尔的抗病毒 方案在 SARS-CoV-2 肺炎患者中安全性良好。

方 法: 回顾性分析了 52 例 SARS-CoV-2 肺炎患者的临 床资料, 分析患者入院时以及抗病毒治疗期间症 状、肝功能及血脂水平等的变化。

结 论: 该方案最常见的不良反应为消化道症状和血脂代 谢异常, 血清甘油三酯、总胆固醇和低密度脂蛋 白胆固醇水平均较治疗前显著升高; 与洛匹那韦/ 利托那韦相比, 达芦那韦/考比司他对血脂代谢的 负面影响较小; 建议对使用蛋白酶抑制剂联合阿 比多尔抗病毒方案的患者密切监测和随访血脂 水平变化。

关键词: 严重急性呼吸综合征冠状病毒 2(SARS-CoV-2); 洛匹那韦; 达芦那韦; 肺炎; 血脂代谢 